\title{
The Chow ring of the moduli space of curves of genus six
}

\author{
Nikola Penev and Ravi Vakil
}

\begin{abstract}
We determine the Chow ring (with $\mathbb{Q}$-coefficients) of $\mathcal{M}_{6}$ by showing that all Chow classes are tautological. (In particular, all algebraic cohomology is tautological, and the natural map from Chow to cohomology is injective.) We stratify the moduli space into locally closed strata which are group quotients, and use a theorem of Vistoli to show that their Chow rings are generated by Chern classes of natural vector bundles. To demonstrate the utility of these methods, we also give quick derivations of the Chow groups of moduli spaces of curves of lower genus. The genus six case relies in addition on the particularly beautiful Brill-Noether theory in this case, and in particular on a rank five vector bundle "relativizing" a baby case of a celebrated construction of Mukai, which we interpret as a subbundle of the rank six vector bundle of quadrics cutting out the canonical curve.
\end{abstract}

\section{Introduction}

Modern progress in our algebro-geometric understanding of the moduli space of smooth curves was launched by Faber's papers [Fab90a, Fab90b] on the Chow rings of $\mathcal{M}_{3}$ and $\mathcal{M}_{4}$, which described tools for understanding about Chow rings of $\mathcal{M}_{g}$, and Faber's conjecture [Fab99, Conjecture 1], which suggested the existence of a rich structure in the "tautological" part of the Chow ring $R^{*}\left(\mathcal{M}_{g}\right) \subset A^{*}\left(\mathcal{M}_{g}\right)$. (Important note: throughout this paper, Chow groups are taken with $\mathbb{Q}$-coefficients.) Earlier work of Mumford [Mum83], and Witten's conjecture [Wit91], provided the foundations for these papers.

Mumford [Mum83, $\S 8$, p. 318] earlier described the Chow ring of $\mathcal{M}_{2}$, and Izadi [Iza95] later determined the Chow ring of $\mathcal{M}_{5}$. In genus up to five the Chow ring is all tautological, and of the form

$$
A^{*}\left(\mathcal{M}_{g}\right)=\mathbb{Q}\left[\kappa_{1}\right] /\left(\kappa_{1}^{g-1}\right) .
$$

In this paper, we describe the Chow ring of $\mathcal{M}_{6}$.

Main Theorem 1.1. We have

$$
A^{*}\left(\mathcal{M}_{6}\right)=\mathbb{Q}\left[\kappa_{1}, \kappa_{2}\right] /\left(127 \kappa_{1}^{3}-2304 \kappa_{1} \kappa_{2}, 113 \kappa_{1}^{4}-36864 \kappa_{2}^{2}\right) .
$$

Received 22 August 2013, accepted in final form 19 September 2014.

2010 Mathematics Subject Classification 14H10, 14C15 (primary), 14H51 (secondary)

Keywords: moduli, curves, Chow ring, intersection theory, Mukai, Brill-Noether theory.

This journal is (C) Foundation Compositio Mathematica 2015. This article is distributed with Open Access under the terms of the Creative Commons Attribution Non-Commercial License, which permits non-commercial reuse, distribution, and reproduction in any medium, provided that the original work is properly cited. For commercial re-use, please contact the Foundation Compositio Mathematica.

The second author was supported by NSF grants DMS-1100771 and DMS-1159156, and the Simons Foundation. 


\section{Nikola Penev and Ravi Vakil}

In particular, in $H^{*}\left(\mathcal{M}_{6}, \mathbb{Q}\right)$, all "algebraic cohomology" is tautological, and the natural map $A^{*}\left(\mathcal{M}_{6}\right) \rightarrow H^{2 *}\left(\mathcal{M}_{6}, \mathbb{Q}\right)$ is an injection.

The explicit description (1.2) of the ring hides its elegance. It should be understood as follows. Faber's conjecture [Fab99] describes one ring for each genus $g$, determined by the fact that it is a Poincaré duality ring of dimension $g-2$, with known generators, and relations determined by the top intersections of these generators, which in turn have a beautiful description in terms of the symmetric group. This ring agrees with the tautological ring in genus up to $g=23$. The case under consideration here is the first genus in which the ring is not of the form (1.1) (see [Fab99, $\S 4$, p. 123]).

Theorem 1.1 is proved in $\S 4$ and $\S 6$; we make the argument explicit in $\S 4.4$.

1.2. Ingredients. We briefly describe the new points of view which make Theorem 1.1 possible.

(i) We take advantage of the fact that Faber has already determined the tautological ring $[$ Fab99, $\S 4$, p. 123]; we will show that all Chow classes are tautological, and need not worry about determining relations.

(ii) We cut up $\mathcal{M}_{6}$ into locally closed strata as is traditional, but we do not bother computing the Chow groups of the strata. Instead, we choose strata which are group quotients, and use a versatile theorem of Vistoli (Theorem 2.3) to show that the Chow rings are generated by Chern classes of some natural vector bundle(s). (Vistoli's theorem was used earlier in this situation by Fontanari and Looijenga; see [FL08, §4, p. 141].) We then show that the fundamental class of the stratum is tautological, and also relate the Chern classes of the vector bundle(s) to the Hodge bundle to show that they too are restrictions of tautological classes on $\mathcal{M}_{6}$.

(iii) For a large open subset $\mathcal{M}_{6}^{M}$ of $\mathcal{M}_{6}$ (the "Mukai-general locus", the complement of the trigonal, hyperelliptic, and plane quintic loci), we use Mukai's work describing the corresponding canonical curves as complete intersections in $G(2,5)$, and in particular we construct a corresponding rank five vector bundle $\mathcal{V}$ on this open subset. Several aspects of Mukai's construction come into play.

The fact that it is tractable to determine $A^{*}\left(\mathcal{M}_{6}\right)$ requires a number of fortunate coincidences. But we hope that our methods will be useful in other circumstances. As a first example, it seems plausible that such methods can show that $A^{*}\left(\mathcal{M}_{g}\right)$ is finitely generated for $7 \leqslant g \leqslant 9$, using Mukai's description of large open subsets of $\mathcal{M}_{g}$ in this genus range. (There seems no reason to believe that $A^{*}\left(\mathcal{M}_{g}\right)$ is finitely generated in general.)

Example 1.3. For those less familiar with the tautological ring, we point out as an example the unusual way in which classes are shown to be zero. We explain how we know that the fundamental class of the locus $\mathcal{B}_{6}$ of bi-elliptic curves (those curves admitting a double cover of an elliptic curve) is 0 . (The difficulty in showing this fact obstructed an earlier attempt by the authors to prove the Main Theorem 1.1.) Even the fact that $\left[\mathcal{B}_{6}\right]$ is 0 in cohomology is not clear; this is the first in a series of interesting questions on the cohomology of $\mathcal{A}_{g}$ (S. Grushevsky, private communication).

The vanishing of $\left[\mathcal{B}_{6}\right]$ is for the following unusual reasons (which are most unlikely to generalize).

The locus $\mathcal{B}_{6}$ is irreducible of codimension five. By Looijenga's Theorem (the main result of [Loo95], see $\S 2.4(\mathrm{i})), R^{5}\left(\mathcal{M}_{6}\right)=0$, so it suffices to show that $\left[\mathcal{B}_{6}\right]$ is tautological. This class lies in the open subset $\mathcal{M}_{6}^{M}$ of Mukai-general curves (see Theorem 5.1). We describe $\mathcal{M}_{6}^{M}$ as a quotient 


\section{The Chow Ring of The Moduli SPACE of CURVES of Genus SIX}

by $G L(5)$ (Theorem 5.6). The dividend is a hypersurface complement in an affine bundle over a Grassmannian. The Chow groups of the quotient are generated, in a suitable sense, by the Chow groups of the Grassmannian and the Chern classes of the vector bundle corresponding to the $G L(5)$-quotient (Proposition 6.2). These can be related to each other, and the Hodge bundle, to show they are all tautological. Thus $\left[\mathcal{B}_{6}\right]$ is forced to be tautological, and hence 0 .

1.4. Structure of paper. In $\S 2$, we collect the tools we will use. In $\S 3$, we demonstrate the approach, by quickly recovering the Chow rings of $\mathcal{M}_{2}$ through $\mathcal{M}_{5}$. In $\S 4$, we give background on $\mathcal{M}_{6}$, mentioning in particular the facts we need. In $\S 4.5$, we show that all classes supported on the trigonal, hyperelliptic, or plane quintic locus of $\mathcal{M}_{6}$ are tautological. In $\S 5$, we describe the rank five "Mukai" bundle on the Mukai-general locus $\mathcal{M}_{6}^{M}$. Finally, in $\S 6$, we show that all Chow classes supported on the Mukai-general locus are tautological. This concludes the proof of Theorem 1.1

\section{Background}

We work over $\mathbb{C}$ for convenience. All quotients should be interpreted as stacks, not coarse moduli spaces. If $V$ is a vector space, then $\mathbb{P} V$ is the projectivization in the sense of Grothendieck, that is, the space of one-dimensional quotients of $V$. Thus $V$ is the space of "linear functionals" on $\mathbb{P} V$. We work throughout with finite type schemes or Deligne-Mumford stacks. The tautological ring $R^{*}\left(\mathcal{M}_{g}\right)$ is the subring of $A^{*}\left(\mathcal{M}_{g}\right)$ (not, a priori, $H^{*}\left(\mathcal{M}_{g}, \mathbb{Q}\right)$ ). If $U$ is an open substack of $\mathcal{M}_{g}$, then $R^{*}(U)$ is defined as $\left.R^{*}\left(\mathcal{M}_{g}\right)\right|_{U}$.

\subsection{Background on Chow groups}

We say that an irreducible variety $X$ is Chow-free if $A_{*} X$ is generated (as a $\mathbb{Q}$-vector space) by the fundamental class $[X]$. If $Z \hookrightarrow X$ is a closed embedding with complement $U$, then we have an exact sequence of Chow groups

$$
A_{*} Z \rightarrow A_{*} X \rightarrow A_{*} U \rightarrow 0
$$

(see [Ful84, Proposition 1.8] for schemes and [Kre99, Proposition 2.3.6] for the extension to stacks). Thus if $D \subset \mathbb{P}^{n}$ is a closed subset containing a (nonempty) hypersurface, then $\mathbb{P}^{n} \backslash D$ is Chow-free. Also, to show that Chow groups of $\mathcal{M}_{g}$ are tautological, it suffices to stratify $\mathcal{M}_{g}$, and to inductively show that the pushforwards of Chow groups of the closures of these strata are all tautological.

For the remainder of $\S 2.1$, suppose that $\pi: X \rightarrow Y$ is a morphism.

Remark 2.2. (i) If $\pi$ is finite and flat of degree $d$, then $\pi^{*}: A_{*} Y \hookrightarrow A_{*} X$ is an injection, as $\frac{1}{d} \pi_{*}$ is a one-sided inverse.

(ii) It is straightforward to show that if $\pi$ is a gerbe banded by a finite group, then $\pi^{*}$ is an isomorphism. (The cycles on $X$ and on $Y$ are obviously identified.)

Theorem 2.3 (Vistoli). Suppose $G=G L$ or $S L$, and that $\pi$ is a quotient by $G$ (that is, $Y=X / G$ categorically). Let $V$ be the corresponding vector bundle on $Y$; if $G=S L$, then $c_{1}(V)=0$. Then:

(i) The pullback $\pi^{*}: A_{*} Y \rightarrow A_{*} X$ is surjective.

(ii) The kernel of $\pi^{*}$ is generated as a group by classes of the form $c_{i}(V) \cap Z$, where $i>0$, and $Z \in A_{*}(Y)$. 


\section{Nikola Penev and Ravi Vakil}

(iii) If $X$ (and hence $Y$ ) is smooth, then $A^{*} Y$ is generated as a ring by $c_{1}(V), \ldots, c_{n}(V)$ and (any chosen) lifts of generators of $A^{*} X$. In particular, if $X$ is Chow-free, then $A^{*} Y$ is generated by $\left\{c_{i}(V)\right\}$.

Parts (i) and (ii) are [Vis87, Theorem 2], and part (iii) follows by induction on codimension.

\subsection{Background on the tautological ring}

(i) Let $\mathcal{H}_{g} \subset \mathcal{M}_{g}$ be the locus of hyperelliptic curves. We will use the following, from the main result of [Loo95]: $R^{i}\left(\mathcal{M}_{g}\right)=0$ for $i>g-2$, and $R^{g-2}\left(\mathcal{M}_{g}\right)$ is generated by [ $\left.\mathcal{H}_{g}\right]$. (In fact, $\left[\mathcal{H}_{g}\right] \neq 0$, [Fab97], but we will not need this.)

(ii) If a Brill-Noether locus is of the expected dimension, then its fundamental class is tautological. (The cases of interest to us here are the loci of hyperelliptic curves, trigonal curves, and plane quintics.) This was shown in [Fab99, $\S 3$, p. 116-118], by suitably describing each Brill-Noether locus as a degeneracy locus.

(iii) We have Pic $\mathcal{M}_{g} \otimes_{\mathbb{Z}} \mathbb{Q}=\mathbb{Q}[\lambda]$. This follows from the section determining Pic $\overline{\mathcal{M}}_{g}$ in [Mum77]. (In the genera of interest to us, $g \leqslant 6$, purely algebraic arguments are possible. For example, in the case $g=6$, an argument is given in the proof of Proposition 4.3.)

\section{Moduli of curves of small Clifford index}

In this section, we show that Chow groups of various classes pushed forward from moduli spaces of curves of low Clifford index are tautological. We first discuss hyperelliptic and trigonal curves. We then deal with low genus curves, partially to show the utility of the methods. The trigonal case is the main substantive part of this section.

\subsection{Hyperelliptic curves}

This discussion can be done simply in other ways, but we deliberately use the same methods as we use later. Fix a genus $g \geqslant 2$. Recall that $\left[\mathcal{H}_{g}\right]$ is tautological, by $\S 2.4(\mathrm{i})$ (Looijenga's theorem) or $\S 2.4$ (ii). Thus to show that the pushforwards of the Chow groups of $\mathcal{H}_{g}$ to $\mathcal{M}_{g}$ are tautological, it suffices to show that the Chow ring of $\mathcal{H}_{g}$ is generated by elements that are pulled back from elements of $R^{*}\left(\mathcal{M}_{g}\right)$.

Let $\mathcal{H}_{g}^{\prime}$ be the moduli space of $2 g+2$ unordered distinct points on a genus zero smooth curve. Then there is a natural map $\rho: \mathcal{H}_{g} \rightarrow \mathcal{H}_{g}^{\prime}$, which is a $\mu_{2}$-gerbe. The pullback map $\rho^{*}: A^{*}\left(\mathcal{H}_{g}^{\prime}\right) \rightarrow A^{*}\left(\mathcal{H}_{g}\right)$ is an isomorphism, by $\S 2.2(\mathrm{ii})$. Thus it suffices to show that the Chow ring of $A^{*}\left(\mathcal{H}_{g}^{\prime}\right)$ is tautological, that is, its elements pull back to tautological elements of $\mathcal{H}_{g}$. Now $\mathcal{H}_{g}^{\prime}=\left(\mathbb{C}^{2 g+3} \backslash \Delta\right) / G L(2)$, where $\mathbb{C}^{2 g+3}$ parametrizes degree $2 g+2$ homogeneous polynomials in $\mathbb{C}[x, y]$, and $\Delta$ is the discriminant locus. The $G L(2)$-quotient induces a vector bundle $\mathcal{W}$ on $\mathcal{H}_{g}^{\prime}$. By Theorem 2.3(iii), as $\mathbb{C}^{2 g+3} \backslash \Delta$ is Chow-free, $A^{*}\left(\mathcal{H}_{g}^{\prime}\right)$ is generated by $c_{1}(\mathcal{W})$ and $c_{2}(\mathcal{W})$. Now $\rho^{*}\left(\mathbb{P} \operatorname{Sym}^{g-1} \mathcal{W}\right)$ is identified with the target of the canonical embedding $\mathbb{P E}$ (where $\mathbb{E}$ is the Hodge bundle), so $\mathrm{Sym}^{g-1} \mathcal{W}$ is isomorphic to $\mathbb{E} \otimes \mathcal{L}$ for some invertible sheaf $\mathcal{L}$ on $\mathcal{H}_{g}$. But $\operatorname{Pic}_{\mathbb{Q}} \mathcal{H}_{g}=0$. (This is well known, and can even be shown by the methods described here; see [AV04, Theorem 5.1] for a much more general statement.) Thus $\mathrm{Sym}^{g-1} \mathcal{W} \cong \mathbb{E}$, so $\mathrm{Sym}^{g-1} \mathcal{W}$ has tautological Chern classes in $A^{*}\left(\mathcal{H}_{g}\right)$, from which (by considering Chern roots) we conclude that $\mathcal{W}$ does as well.

Thus the pushforwards of the Chow groups of $\mathcal{H}_{g}$ are all tautological. (In particular, by $\S 2.4(\mathrm{i})$, the pushforward yields just multiples of the fundamental class $\left[\mathcal{H}_{g}\right]$ in $A^{*}\left(\mathcal{M}_{g}\right)$.) 


\section{The Chow Ring of the MOduli SPACE of CURVES OF Genus SIX}

\subsection{Trigonal curves}

It would be good to know that the Chow groups of the locus of trigonal curves are tautological in $\mathcal{M}_{g}$. We will establish something weaker. See Remark 3.4 for what is missing.

Fix a genus $g \geqslant 4$. Let $\mathcal{T}_{g}$ be the stack of smooth genus $g$ curves, along with the data of a triple cover of a genus zero curve. If $g>4$, the natural moduli morphism $\mathcal{T}_{g} \rightarrow \mathcal{M}_{g}$ is a closed embedding. (This is well known and straightforward to show; simply describe an equivalence of categories between $\mathcal{T}_{g}$ and a closed substack of $\mathcal{M}_{g}$. See [BV12, Proposition 2.2] for details, and much more.)

Recall that $\mathcal{T}_{g}$ is stratified by Maroni invariant. The Maroni invariant of a triple cover $\pi$ : $C \rightarrow \mathbb{P}^{1}$ is the unique nonnegative integer $n$ such that $\pi$ factors through a closed embedding $\pi: C \hookrightarrow \mathbb{F}_{n}$ where $\mathbb{F}_{n}$ is the Hirzebruch surface $\mathbb{P}_{\mathbb{P}^{1}}(\mathcal{O} \oplus \mathcal{O}(n))$ (with the implicit projection to $\mathbb{P}^{1}$ as the trigonal structure morphism). We denote the "standard" elements of $A^{1}\left(\mathbb{F}_{n}\right)$ by $E$ (the class of a section of self-intersection $-n$, unique if $n>0$ ), $S$ (the class of a section not meeting $E$, of self-intersection $n$ ), and $F$ (the fiber class). Important note: we will use $S$ to mean an actual choice of section, not just a Chow class.

Then $C \hookrightarrow \mathbb{F}_{n}$ is in class $3 S+k F$, where $k=(g-3 n+2) / 2$. In particular, $n$ must have the same parity as $g$, and $n \leqslant(g+2) / 3$.

TheOREM 3.3. If $\mathcal{T}_{g, n}$ is the space of trigonal genus $g$ smooth curves of Maroni invariant $n$, then any Chow class on $\mathcal{T}_{g, n}$ is the pullback of a tautological class on $\mathcal{M}_{g}$.

Remark 3.4. If we knew that the fundamental classes $\left[\mathcal{T}_{g, n}\right]$ were also tautological on $\mathcal{M}_{g}$ for all $n$, we would then know that the (pushforward of the) Chow groups of the locus of all trigonal curves are tautological in $\mathcal{M}_{g}$.

Proof of Theorem 3.3.

Case $n>0$.

Consider $\mathbb{P}^{N}:=\mathbb{P} H^{0}\left(\mathbb{F}_{n}, \mathcal{O}(3 S+k F)\right)$, where $k=(g-3 n+2) / 2$. Let $\Delta$ be the divisor on $\mathbb{P}^{N}$ corresponding to singular curves. (Note that $\mathbb{P}^{N} \backslash \Delta$ is Chow-free.)

Now $\mathcal{T}_{g, n} \cong\left(\mathbb{P}^{N} \backslash \Delta\right) /$ Aut $\mathbb{F}_{n}$. Our goal is to show that the Chow groups of this quotient are all restrictions of tautological classes.

Let $G \subset$ Aut $\mathbb{F}_{n}$ be the subgroup fixing the curve $S$ as a set (but not necessarily pointwise). Then $\left(\mathbb{P}^{N} \backslash \Delta\right) / G \rightarrow \mathcal{T}_{g, n}$ is an $\mathbb{A}^{n+1}$-bundle (as $h^{0}\left(\mathbb{F}_{n}, \mathcal{O}(S)\right)=n+2$ ), so pullback by this morphism induces an isomorphism of Chow rings by the homotopy axiom of [Kre99, Corollary 2.5.7]. Hence it suffices to show that the Chow groups of $\left(\mathbb{P}^{N} \backslash \Delta\right) / G$ are restrictions of tautological classes.

We have a filtration

$$
1 \rightarrow \mathbb{G}_{m} \rightarrow G \rightarrow \operatorname{Aut}\left(\mathbb{P}^{1}\right) \rightarrow 1
$$

where the $\mathbb{G}_{m}$ acts on $\mathbb{F}_{n}$ by its action on the fibers (fixing both $E$ and $S$ ), and Aut $\left(\mathbb{P}^{1}\right.$ ) is the automorphism group of the target $\mathbb{P}^{1}$. We extend $G$ to a group $G^{\prime}$ along the surjection $S L(2) \rightarrow \operatorname{Aut}\left(\mathbb{P}^{1}\right)=P G L(2):$

$$
1 \rightarrow \mathbb{G}_{m} \rightarrow G^{\prime} \rightarrow S L(2) \rightarrow 1 .
$$

Then $\left(\mathbb{P}^{N} \backslash \Delta\right) / G^{\prime} \rightarrow\left(\mathbb{P}^{N} \backslash \Delta\right) / G$ is a $\mu_{2}$-gerbe, so pullback by this morphism induces an isomorphism of Chow groups by $\S 2.2$ (ii). Hence it suffices to show that the Chow groups of $\left(\mathbb{P}^{N} \backslash \Delta\right) / G^{\prime}$ are restrictions of tautological classes. 


\section{Nikola Penev and Ravi Vakil}

By Theorem 2.3(iii), the Chow groups of this quotient $\left(\mathbb{P}^{N} \backslash \Delta\right) / G^{\prime}$ will be generated by $\alpha_{1}$, which is defined to be the first Chern class of the line bundle $\mathcal{L}$ corresponding to $\mathbb{G}_{m}$, and $\beta_{2}$, which is defined to be the second Chern class of the vector bundle $V$ corresponding to the $S L(2)$. (Here we use $c_{1}(V)=0$.) In particular, the only line bundles on the quotient $\left(\mathbb{P}^{N} \backslash \Delta\right) / G^{\prime}$ are $\mathbb{Q}$-multiples of $\alpha_{1}$.

Geometrically, $V$ should be interpreted as a vector space such that $\mathbb{P} V$ is the target $\mathbb{P}^{1}$, and $\mathcal{L}$ can be informally interpreted as a fiber of $\mathbb{F}_{n} \backslash E$ over any point of the target $\mathbb{P}^{1}$ (with the intersection with $S$ as 0 ).

We now relate these two bundles to the Hodge bundle $\mathbb{E}$. We motivate this with some geometry. If we canonically embed the trigonal curve in $\mathbb{P}^{g-1}$, the ambient Hirzebruch surface "comes along for the ride" as a scroll. Geometrically: the fibers of the $\mathbb{P}^{1}$ are recovered from the fact that the "trigonally conjugate points" are collinear in the canonical embedding. Sheaf-theoretically, this is the fact that if $D$ is such a trigonal curve on $\mathbb{F}_{n}$, then

$$
H^{0}\left(\mathbb{F}_{n}, \mathcal{O}(S+(n+k-2) F)\right) \rightarrow H^{0}\left(D, \mathcal{O}_{D}(S+(n+k-2) F)\right)
$$

is an isomorphism, and the right-hand side is canonically identified with the differentials on $D$.

Better yet, since we have a privileged curve $S$ on $\mathbb{F}_{n}$ (not just a curve class), we have a canonical direct sum decomposition

$$
H^{0}\left(\mathbb{F}_{n}, \mathcal{O}(S+(n+k-2) F)\right)=H^{0}(S, \mathcal{O}(2 n+k-2)) \oplus H^{0}(E, \mathcal{O}(n+k-2))
$$

which we interpret geometrically as follows. We have that $\mathbb{F}_{n}$ is embedded in $\mathbb{P}^{g-1}, E$ is a degree $n+k-2$ rational normal curve, $S$ is a degree $2 n+k-2$ rational normal curve, the projective spaces they span have empty intersection, and together they span the full $\mathbb{P}^{g-1}$.

Let $\mathbb{P}^{1}=\mathbb{P} V$, where $V$ is a two-dimensional vector space, and let $\mathcal{L}$ be the one-dimensional tautological representation of $\mathbb{G}_{m}$. Then the canonical embedding is given by

$$
\left(\operatorname{Sym}^{2 n+k-2} V\right) \oplus\left(\mathcal{L} \otimes \operatorname{Sym}^{n+k-2} V\right) \text {. }
$$

Here $\beta_{2}=c_{2}(V)$, and $\alpha_{1}=c_{1}(\mathcal{L})$. Since we have identified the projectivization of this bundle (3.1) with $\mathbb{P E}$, the Hodge bundle $\mathbb{E}$ must differ from this bundle (3.1) by some line bundle $\mathcal{M}$ :

$$
\mathbb{E} \otimes \mathcal{M} \cong\left(\operatorname{Sym}^{2 n+k-2} V\right) \oplus\left(\mathcal{L} \otimes \operatorname{Sym}^{n+k-2} V\right) .
$$

As mentioned above, $\mathcal{M}$ must be some $\mathbb{Q}$-multiple of $\mathcal{L}$ (that is, $c_{1}(\mathcal{M})$ is some multiple of $\alpha_{1}$ ). Comparing the first Chern class of both sides, we see that $\mathcal{L}$ is some multiple of $\lambda_{1}$. Comparing the second Chern class of both sides, we see that $\beta_{2}$ is a linear combination of $\lambda_{1}^{2}$ and $\lambda_{2}$. Case $n=0$.

By [Sta00, Theorem IV], Pic $\mathcal{T}_{g, 0} \otimes_{\mathbb{Z}} \mathbb{Q}=0$. Consider the finite flat degree $2 g+4$ cover $\mathcal{X} \rightarrow \mathcal{T}_{g, 0}$, parametrizing genus $g$ trigonal covers along with the choice of a branch point of the cover.

Fix a point $p=(\infty, \infty) \in \mathbb{P}^{1} \times \mathbb{P}^{1}=\mathbb{F}_{0}$. Let $\mathcal{I}$ be the ideal sheaf (on $\mathbb{F}_{0}$ ) of a first-order thickening of $p$ in the "vertical" direction.

Consider $\mathbb{P}^{N}:=\mathbb{P} H^{0}\left(\mathbb{F}_{0}, \mathcal{I}(3 S+k F)\right)$, where $k=(g+2) / 2$. Let $\Delta$ be the divisor on $\mathbb{P}^{N}$ corresponding to singular curves. (Note that $\mathbb{P}^{N} \backslash \Delta$ is Chow-free.)

Now $\mathcal{X} \cong\left(\mathbb{P}^{N} \backslash \Delta\right) /\left(\right.$ Aut $\mathbb{A}^{1} \times$ Aut $\mathbb{A}^{1}$ ) (where the $\mathbb{A}^{1}$ 's are identified with the horizontal and vertical fibers through $p$, but excluding $p$ ). From the exact sequence

$$
0 \rightarrow \mathbb{A}^{1} \rightarrow \text { Aut } \mathbb{A}^{1} \rightarrow \mathbb{G}_{m} \rightarrow 0
$$




\section{The Chow Ring of The Moduli SPACE of CURVES of Genus SIX}

and the fact that $\mathbb{A}^{1}$-bundles induce isomorphisms on Chow rings (by the homotopy axiom of [Kre99, Corollary 2.5.7]), we conclude (from Theorem 2.3(iii)) that $A^{*}(\mathcal{X})$ is generated by the first Chern classes of the two line bundles which are identified with the horizontal and vertical directions. But these line bundles are pulled back from $\mathcal{T}_{g, 0}$, and are thus (rationally) trivial. Hence $A^{*}(\mathcal{X})=0$, and so by Remark $2.2(\mathrm{i}), A^{*}\left(\mathcal{T}_{g, 0}\right)=0$.

\subsection{Curves of low genus}

We now apply these methods to show that $A^{*}\left(\mathcal{M}_{g}\right)$ is tautological for $g<6$. The case $g=2$ is a special case of our hyperelliptic discussion, $\S 3.1$.

\subsubsection{Genus three}

We stratify $\mathcal{M}_{3}$ into the nonhyperelliptic locus $\mathcal{M}_{3} \backslash \mathcal{H}_{3}$ and the hyperelliptic locus $\mathcal{H}_{3}$. We established in $\S 3.1$ that all Chow groups of $\mathcal{H}_{3}$ are tautological (and indeed that $\mathcal{H}_{3}$ is Chow-free), so we concentrate on the open stratum $\mathcal{M}_{3} \backslash \mathcal{H}_{3}$, which parametrizes smooth plane quartics up to automorphisms of the plane. Hence $\mathcal{M}_{3} \backslash \mathcal{H}_{3} \cong\left(H^{0}\left(\mathbb{P}^{2}, \mathcal{O}(4)\right) \backslash \Delta\right) / G L(3)$. The vector bundle corresponding to the $G L(3)$-quotient is the Hodge bundle. Thus the Chow ring of $\mathcal{M}_{3} \backslash \mathcal{H}_{3}$ is generated by the Chern classes of the Hodge bundle, so $A^{*}\left(\mathcal{M}_{3} \backslash \mathcal{H}_{3}\right)$ is all tautological.

\subsubsection{Genus four}

We cut $\mathcal{M}_{4}$ into three strata:

$\mathcal{M}_{4}^{0}$ (dense, dimension 9): those curves whose canonical models are the intersections of smooth quadrics and cubics.

This has a double cover (that is, degree two finite flat cover) $\mathcal{M} \rightarrow \mathcal{M}_{4}^{0}$ corresponding to such curves with a choice of one of the two $g_{3}^{1}$ 's. Then by our trigonal discussion $\S 3.2, A^{*}(\mathcal{M})$ is generated by tautological classes. Hence by $\S 2.2(\mathrm{i}), A^{*}\left(\mathcal{M}_{4}^{0}\right)$ is all tautological.

$\mathcal{T}_{g, 2}$ (dimension 8): those curves whose canonical models are intersections of cones and cubics (or: the divisor of curves with a vanishing theta-null).

We first note that $\left[\mathcal{T}_{g, 2}\right] \in A^{1}\left(\mathcal{M}_{4}\right)$ is tautological, by $\S 2.4($ iii). (As noted there, this can be shown in many purely algebraic ways.) We are then done by Theorem 3.3.

$\mathcal{H}_{4}$ : The hyperelliptic locus (dimension 7 ).

This was dealt with in $\S 3.1$.

\subsubsection{Genus five}

We cut $\mathcal{M}_{5}$ into three strata:

$\mathcal{M}_{5}^{0}$ (dense, dimension 12): non-trigonal non-hyperelliptic curves.

These are the curves whose canonical models are complete intersections of three quadrics in $\mathbb{P}^{4}$. We consider $G(3,15)$ parametrizing nets (linearly embedded planes $\mathbb{P}^{2}$ ) of quadrics in $\mathbb{P}^{4}$. This comes with a rank three bundle, the tautological bundle $\mathcal{Q}$ of the Grassmannian. Let $\Delta$ be the divisor on $G(3,15)$ corresponding to nets of quadrics whose intersection is not a smooth curve. Then $(G(3,15) \backslash \Delta) / P G L(5) \cong \mathcal{M}_{5}^{0}$ (we have a equivalence of moduli problems). We then consider $(G(3,15) \backslash \Delta) / S L(5)$. This is a $\mu_{5}$-gerbe over $(G(3,15) \backslash \Delta) / P G L(5) \cong \mathcal{M}_{5}^{0}$, and thus has the same rational Chow groups by $\S 2.2$ (ii).

Let $\mathcal{V}$ be the tautological rank five vector bundle on the quotient corresponding to the $S L(5)$ bundle. By Theorem 2.3, the Chow classes of the quotient are generated by (lifts of) the coho- 


\section{Nikola Penev and Ravi Vakil}

mology classes on $G(3,15)$, and the Chern classes of $\mathcal{V}$. Recall that the Chow ring of $G(k, n)$ is generated by the Chern classes of the tautological bundle (see for example [Ful84, § 14.7]).

Now $\mathbb{P E} \cong \mathbb{P} \mathcal{V}$ as projective bundles over $\mathcal{M}_{5}^{0}$, as the universal curve is embedded canonically in both projective bundles. Thus $\mathcal{V} \otimes \mathcal{L} \cong \mathbb{E}$, for some line bundle $\mathcal{L}$ on $\mathcal{M}_{5}^{0}$. As $\operatorname{det} \mathcal{V}=\mathcal{O}, \mathcal{L}$ is a (canonical) fifth root of $\operatorname{det} \mathbb{E}$. (Hence $c_{1}(\mathcal{L})=\lambda_{1} / 5$ in $A^{1}((G(3,15) \backslash \Delta) / S L(5))$.) Now $\mathcal{Q}$ is a rank three subbundle of $\operatorname{Sym}^{2} \mathcal{V}$, so $\mathcal{Q} \otimes \mathcal{L}^{\otimes 2}$ is a subbundle of $\mathrm{Sym}^{2} \mathbb{E}$. This bundle $\mathcal{Q} \otimes \mathcal{L}^{\otimes 2}$ is the kernel of the surjective map of vector bundles $\operatorname{Sym}^{2} \pi_{*} \omega \rightarrow \pi_{*} \omega^{\otimes 2}$, where $\pi: \mathcal{C}_{5}^{0} \rightarrow \mathcal{M}_{5}^{0}$ is the universal curve, and $\omega$ is the relative dualizing sheaf. The Chern classes of $\operatorname{Sym}^{2} \pi_{*} \omega=\mathrm{Sym}^{2} \mathbb{E}$ are tautological, and the Chern classes of $\pi_{*} \omega^{\otimes 2}$ are as well (by Grothendieck-Riemann-Roch, see for example [Fab99, $\S 2 \mathrm{a}$, p. 111]). Thus the Chern classes of $\mathcal{Q}$ are also tautological.

In conclusion, the Chow classes of the quotient are thus tautological, because they arise from (lifts of) the cohomology classes on $G(3,15)$ (which are all tautological), and the Chern classes of the $\mathcal{V} \cong \mathbb{E} \otimes \mathcal{L}^{\vee}$.

$\mathcal{T}_{3,1}$ (dimension 11): trigonal curves.

All genus five trigonal curves have Maroni invariant one. As $\left[\mathcal{T}_{3,1}\right] \in A^{*}\left(\mathcal{M}_{5}\right)$ is tautological by $\S 2.4($ ii), we are done by Remark 3.4.

$\mathcal{H}_{5} P($ dimension 9$)$ : hyperelliptic curves.

This was dealt with in $\S 3.1$.

\section{Stratification of $\mathcal{M}_{6}$, and structure of the argument}

The following stratification follows immediately from [ACGH85, Theorem 5.2] (Mumford's refinement of Marten's Theorem), and straightforward dimension counts.

Theorem 4.1. The space $\mathcal{M}_{6}$ may be partitioned into the following locally closed subsets:

(i) (dimension 15) the locus of "Brill-Noether-general" curves, which we denote by $\mathcal{M}_{6}^{B N}$, consisting of curves with finitely many $g_{4}^{1}$ 's;

(ii) (dimension 13) the trigonal locus $\mathcal{T}_{6}$;

(iii) (dimension 12) the locus $\mathcal{Q}_{6}$ of plane quintics;

(iv) (dimension 11) the hyperelliptic locus $\mathcal{H}_{6}$;

(v) (dimension 10) the bi-elliptic locus $\mathcal{B}_{6}$.

Proposition 4.2. The union $\mathcal{M}_{6}^{M}:=\mathcal{M}_{6}^{B N} \cup \mathcal{B}_{6}$ is an open subset of $\mathcal{M}_{6}$. Equivalently, $\mathcal{B}_{6}$ does not meet the closure $\left(\right.$ in $\left.\mathcal{M}_{6}\right)$ of $\mathcal{T}_{6}, \mathcal{Q}_{6}$, or $\mathcal{H}_{6}$.

We call $\mathcal{M}_{6}^{M}$ the Mukai-general locus (for reasons that will be clear by the start of $\S 5$ ), and the corresponding curves Mukai-general curves.

Proof. From the main theorem of [Sch86] (see [Sch86, Table 1]), there are three possible Bettitables for the canonical rings of smooth genus six curves, depending on whether the curve is in $\mathcal{M}_{6}^{M}=\mathcal{M}_{6}^{B N} \cup \mathcal{B}_{6}, \mathcal{T}_{6} \cup \mathcal{Q}_{6}$, or $\mathcal{H}_{6}$. By upper-semicontinuity, from this table the first locus is open.

Alternatively, one could consider $W_{4}^{1}(C)$ as $[C]$ varies over $\mathcal{M}_{6}$. Or one could use the EnriquesPetri Theorem; see [S-D73, Main Theorem].

The following fact is a special case of $\S 2.4($ iii). 


\section{The Chow Ring of The Moduli SPACE of CURVES of Genus SIX}

Proposition 4.3. Pic $\mathcal{M}_{6} \otimes_{\mathbb{Z}} \mathbb{Q}=\mathbb{Q}[\lambda]$.

As promised there, we give a purely algebraic argument.

Proof. Let $\mathcal{M}_{6}^{0}$ be the open subset of $\mathcal{M}_{6}^{B N}$ corresponding to those curves with precisely five $g_{4}^{1}$ 's. It has a degree 120 cover corresponding to such curves with five labeled $g_{4}^{1}$ 's. These in turn are parametrized by a hypersurface complement in $\mathbb{P}^{15}$, parametrizing plane sextic curves, smooth except for nodes at $[1,0,0],[0,1,0],[0,0,1]$, and $[1,1,1]$. This has trivial codimension one rational Chow group, and thus $\mathcal{M}_{6}^{0}$ does too, by Remark 2.2(i). The complement is an irreducible divisor, generically corresponding to a genus six curve with precisely four $g_{4}^{1}$ 's (one with multiplicity two); see [ACGH85, Exercises V.A]. Finally, $\lambda$ is nonzero, for example by using ampleness, or the fact that the tautological ring is known [Fab99, §4, p. 123].

4.4. How we will prove Main Theorem 1.1. As with the examples of $\mathcal{M}_{2}$ through $\mathcal{M}_{5}$ in $\S 3$, we will prove Main Theorem 1.1 by proving that all Chow groups are tautological, and in turn do this by showing that the pushforward of all Chow classes from all strata described in Theorem 4.1 are tautological. We deal with the complement of the Mukai-general locus in the remainder of $\S 4$, and we deal with the Mukai-general locus in the remaining two sections of the paper. By the "excision exact sequence for Chow groups" (2.1), this will complete the proof of Theorem 1.1.

\subsection{Classes pushed forward from the complement of the Mukai-general locus are tautological}

We established in $\S 3.1$ that the (pushforward of) Chow classes on the hyperelliptic locus $\mathcal{H}_{6}$ are tautological.

4.5.1 The plane quintics $\mathcal{Q}_{6}$. By $\S 2.4(\mathrm{ii}),\left[\mathcal{Q}_{6}\right]$ is tautological. As $h^{0}\left(\mathbb{P}^{2}, \mathcal{O}(5)\right)=21$, we may present $\mathcal{Q}_{6}$ as $\left(\mathbb{A}^{21}-\Delta\right) / G L(3)$, where $\Delta$ is the "discriminant" divisor corresponding to singular quintics. Thus the Chow ring of $\mathcal{Q}_{6}$ is generated by the Chern classes of the corresponding rank three vector bundle $V$. But $\operatorname{Sym}^{2} V$ is identified with the Hodge bundle $\left.\mathbb{E}\right|_{\mathcal{Q}_{6}}$ (the canonical bundle of a plane quintic $C$ is canonically identified with the restriction of $\left.\left.\mathcal{O}_{\mathbb{P}^{2}}(2)\right|_{C}\right)$, so we can write the Chern classes of $V$ in terms of the Chern classes $\lambda_{i}$ of the Hodge bundle.

4.5.2 Trigonal curves $\mathcal{T}_{6}$. Similar to the genus four case $(\S 3.5 .2)$, we further stratify $\mathcal{T}_{6}$ by Maroni invariant: it is the union of the Maroni-general locus $\mathcal{T}_{6,0}$ and the "Maroni divisor" $\mathcal{T}_{6,2}$. The first has tautological fundamental class by $\S 2.4(\mathrm{ii})$, and the second does by [Sta00, eq. (1.4)]. Then we are done by Theorem 3.3.

\section{The rank five Mukai bundle on $\mathcal{M}_{6}^{M}$}

Mukai gives a remarkable characterization of "most" canonical curves of genus six through nine in his series of papers starting with [Muk93]. We will rely on his genus six results, given in $[$ Muk93, §5].

Theorem 5.1 (Mukai, [Muk93, §5]). If $C$ is a Mukai-general curve of genus six, then there is a unique stable rank two vector bundle $E$ on $C$ with $\operatorname{det} E \cong \mathcal{K}_{C}$ and having a five-dimensional vector space $V$ of sections. The bundle $E$ is globally generated, and thus yields a morphism 


\section{Nikola Penev and Ravi Vakil}

$C \rightarrow G(2, V)$, which is a closed embedding. This closed embedding is a complete intersection of four linear forms and one quadratic form, under the Plücker embedding $G(2, V) \hookrightarrow \mathbb{P}\left(\wedge^{2} V\right)$.

Caution. When reading [Muk93, $\S 5]$, note that "del Pezzo surfaces" there are not assumed to be smooth. More precisely, if $C$ is a Mukai-general curve, then it may be described as a quadratic section of a dimension two linear complete intersection of $G(2,5)$, and this surface is a del Pezzo surface, possibly singular. For a complete description of the possible surfaces, see [AH81, §5]. For example, in the case of bi-elliptic $C$, it is a cone over an elliptic quintic; see [AH81, p. 169].

Remark 5.2. As $\mathcal{K}_{G(2, V)}=\left.\mathcal{O}_{\mathbb{P} \wedge^{2} V}(-5)\right|_{G(2, V)}$, by the adjunction formula, $\mathcal{K}_{C}=\left.\mathcal{O}_{\mathbb{P} \wedge^{2} V}(1)\right|_{C}$. Let $F$ be the four-dimensional subspace of linear forms of Theorem 5.1, and let $G$ be its sixdimensional quotient:

$$
0 \rightarrow F \rightarrow \wedge^{2} V \rightarrow G \rightarrow 0 .
$$

The space $F$ cuts out $\mathbb{P} G \subset \mathbb{P} \wedge^{2} V$, in which $C$ is nondegenerately embedded. Thus $\mathbb{P} G$ is identified with $\mathbb{P} H^{0}\left(C, \mathcal{K}_{C}\right)$, and thus up to a scalar multiple $G$ is identified with $H^{0}\left(C, \mathcal{K}_{C}\right)$.

Remark 5.3. The vector bundle $E$ has a useful description in terms of the $g_{4}^{1}$ 's of $C$.

TheOREM 5.4. If $\mathcal{L}$ is a line bundle giving a $g_{4}^{1}$, and $\mathcal{M}=\mathcal{K}_{C} \otimes \mathcal{L}^{\vee}$ is its Serre-dual $g_{6}^{2}$, then $E$ may be written in an exact sequence

$$
0 \longrightarrow \mathcal{L} \stackrel{\beta}{\longrightarrow} E \longrightarrow \mathcal{M} \longrightarrow 0
$$

This is the unique (up to scaling) nontrivial extension of $\mathcal{M}$ by $\mathcal{L}$.

The genus eight analog of Theorem 5.4 is shown in [Muk93, Lemma 3.6], and as described in $[$ Muk93, § 5], the analogous argument holds in genus six.

5.5. We will need a refined "family" version of Theorem 5.1, which is undoubtedly known to experts, although we were unable to find a reference. This describes $\mathcal{M}_{6}^{M}$ as a quotient $(Y \backslash \Delta) / G L(5)$. We now define the terms in this quotient.

Let $V$ be a dimension five vector space. Consider the Grassmannian $G(2, V)$, which has its Plücker embedding in $\mathbb{P} \wedge^{2} V$. The four-dimensional vector space of linear sections of $G(2, V)$ (under the Plücker embedding) are thus parametrized by $G\left(4, \wedge^{2} V\right)$.

The quadratic forms on such complete intersections (again, in the Plücker variables) are parametrized by a rank 16 vector bundle on $G\left(4, \wedge^{2} V\right)$ : there is the $\left(\begin{array}{c}5+2 \\ 2\end{array}\right)=21$-dimensional vector space of quadratic forms on the $\mathbb{P}^{5}$ cut out in $\mathbb{P}\left(\wedge^{2} V\right)$ by the four linear forms; but a five-dimensional vector space of them lie in the ideal generated by the Plücker quadrics.

Denote this rank 16 vector bundle over $G\left(4, \wedge^{2} V\right)$ by $Y$. Note that $\operatorname{dim} Y=40$. Then $Y$ parametrizes complete intersections of $G(2, V)$ with four linear forms and a quadratic form. The locus in $Y$ where such complete intersections are singular is a divisor $\Delta$. Then $Y \backslash \Delta$ corresponds to the smooth complete intersections. It is straightforward (see for example [Muk93, $\S 5]$ ) that such complete intersections are canonically embedded (irreducible) genus six curves. The group $G L(V)$ acts on $Y \backslash \Delta$ through its action on $G(2, V)$.

Theorem 5.6. The natural map $\phi:(Y \backslash \Delta) / G L(V) \rightarrow \mathcal{M}_{6}$ is an open embedding (of DeligneMumford stacks) whose image is precisely $\mathcal{M}_{6}^{M}$.

For this reason, we call $\mathcal{M}_{6}^{M}$ the Mukai-general locus. Denote the universal curve over $\mathcal{M}_{6}^{M}$ by $\mathcal{C}^{M} \rightarrow \mathcal{M}_{6}^{M}$. If $[C] \in \mathcal{M}_{6}^{M}$, we say that $C$ is a Mukai-general curve (of genus six). 


\section{The Chow Ring of The Moduli SPACE of CURVES of Genus SIX}

Proof. Note first that the image of $\phi$ is precisely $\mathcal{M}_{6}^{M}$, by Mukai's work. By the Enriques-Petri theorem (see [S-D73, Main Theorem]), these are the only canonical curves cut out by quadrics. Conversely, if a curve $C$ is Mukai-general, then it is in the image of $\phi$ by [Muk93, Theorem 5.2]. To find an inverse image of $C$ in $(Y \backslash \Delta)$, we take the unique stable rank 2 vector bundle $E$ with $\operatorname{det} E=K_{C}$ and $h^{0}(C, E)=5$, which happens to be globally generated (Theorem 5.1), and take the induced map $C \rightarrow G(2,5)$.

We next claim that the map $\phi$ is representable, that is, gives an isomorphism of isotropy groups. The previous paragraph establishes $Y \backslash \Delta$ as representing the moduli space of triples of a curve in the Mukai-general locus $C$, a stable rank two vector bundle $E$ with $\operatorname{det}(E) \cong \mathcal{K}_{C}$ and $h^{0}(C, E)=5$, and a framing of $h^{0}(C, E)$. The quotient by $G L(5)$ forgets the framing. We thus must show that for each curve $C$ in the Mukai-general locus, the vector bundle $E$ is unique up to scaling. This is a slight strengthening of Mukai's result [Muk93, Theorem 5.1(1)], which shows that $E$ is unique up to isomorphism.

We filter $E$ as in (5.1). Hence given any automorphism $\alpha: E \rightarrow E$, we have a diagram

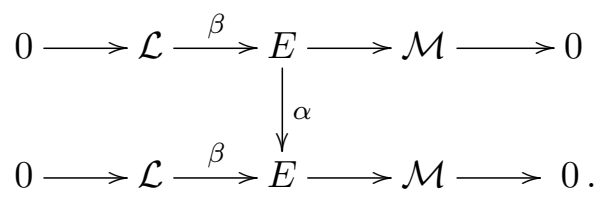

We need the further fact that the inclusions $\mathcal{L} \hookrightarrow E$ are unique up to scaling. For this we need that $\operatorname{Hom}(\mathcal{L}, \mathcal{M})=0$. But $\operatorname{Hom}(\mathcal{L}, \mathcal{M})=H^{0}\left(C, \mathcal{M} \otimes \mathcal{L}^{\vee}\right)$ is 0 , as $\mathcal{M} \otimes \mathcal{L}^{\vee}$ is a line bundle of degree $5-3=2$, and $C$ is not hyperelliptic. Thus after rescaling $\alpha$, we can extend the diagram as follows:

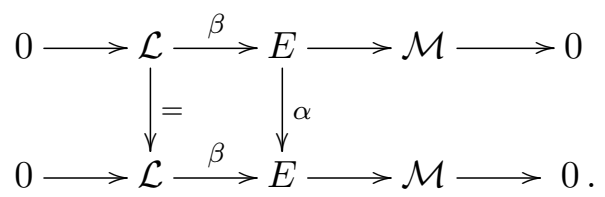

Finally, noting that there is only one nontrivial extension of $\mathcal{M}$ by $\mathcal{L}$ (up to scaling of $\mathcal{L}$ and $\mathcal{M}$ and the extension class in $\operatorname{Ext}^{1}(\mathcal{M}, \mathcal{L})$ ) yielding a vector bundle with $E$ with five sections ([Muk93, Lemma 3.6], translated to the genus six setting as in [Muk93, §5]), after rescaling $\mathcal{M}$ in the bottom row of the diagram above, we may write

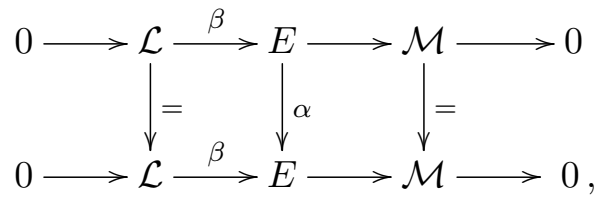

from which we see that $E$ is unique up to scaling, as desired.

We have thus established that $\phi$ is representable. Finally, as $\phi$ is a (representable) birational bijection onto its image $\mathcal{M}_{6}^{M}$, and as $\mathcal{M}_{6}^{M}$ is normal, the map $\phi$ is an isomorphism onto $\mathcal{M}_{6}^{M}$.

Remark 5.7. Just as Mukai's paper [Muk93] treats genus six curves by precisely the same method as genus eight curves, the proof of Theorem 5.6 applies with the obvious changes to the genus eight case.

5.8. The Mukai vector bundle $\mathcal{V}$ on $\mathcal{M}_{6}^{M}$. This argument shows the existence of a rank 5 vector bundle $\mathcal{V}$ on $\mathcal{M}_{6}^{M}$ (and a rank two vector bundle $\mathcal{E}$ on the universal curve $\mathcal{C}^{M}$ over $\mathcal{M}_{6}^{M}$ ), relativizing Mukai's construction. For obvious reasons we call $\mathcal{V}$ the Mukai bundle. 


\section{Nikola Penev and RaVi VAKIL}

By the construction of $\mathcal{M}_{6}^{M}$ of Theorem 5.6, we have a tautological section to $G\left(4, \wedge^{2} \mathcal{V}\right) \rightarrow$ $\mathcal{M}_{6}^{M}$, from which we have a tautological exact sequence of locally free sheaves on $\mathcal{M}_{6}^{M}$ :

$$
0 \rightarrow \mathcal{F} \rightarrow \wedge^{2} \mathcal{V} \rightarrow \mathbb{E}^{\prime} \rightarrow 0,
$$

where $\operatorname{rank} \mathcal{F}=4$ and $\operatorname{rank} \mathbb{E}^{\prime}=6$. We use the notation $\mathbb{E}^{\prime}$ because we may interpret it as a "twist" of the Hodge bundle $\mathbb{E}$, as follows.

PROPOSITION 5.9.

(a) We have $\mathbb{E}^{\prime} \cong \mathbb{E} \otimes \mathcal{L}^{\prime}$ for some invertible sheaf $\mathcal{L}^{\prime}$ on $\mathcal{M}_{6}^{M}$.

(b) The Chern classes of $\mathbb{E}^{\prime}$ are tautological.

Proof. For a point $[C] \in \mathcal{M}_{6}^{M}$ of the Mukai-general locus, the fibers of $\mathcal{F}$ and $\mathbb{E}^{\prime}$ are the vector spaces $F$ and $G$ of $\S 5.2$. In particular, $\mathbb{P E}^{\prime}$ is canonically isomorphic to $\mathbb{P E}$, so that the following diagram commutes:

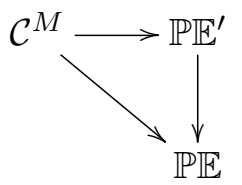

where the vertical map $\mathbb{P E}^{\prime} \rightarrow \mathbb{P E}$ is the (canonical) isomorphism, the diagonal map $\mathcal{C}^{M} \rightarrow \mathbb{P E}$ is the canonical map of $\mathcal{C}^{M}$ into the projectivization of its relative canonical bundle, and the horizontal map $\mathcal{C}^{M} \rightarrow \mathbb{P} \mathbb{E}^{\prime}$ is the map corresponding to the pointwise construction of $\S 5.2$. This implies part (a).

As $\operatorname{Pic}_{\mathbb{Q}} \mathcal{M}_{6}^{M} \equiv \operatorname{Pic}_{\mathbb{Q}} \mathcal{M}_{6}$ (they differ by a codimension two subset, by Theorem 4.1), and Pic $_{\mathbb{Q}} \mathcal{M}_{6}$ is generated by $\lambda$ (Proposition 4.3), we have part (b).

\section{The Mukai-general locus: $A^{*}\left(\mathcal{M}_{6}^{M}\right)$ is tautological}

In this section we complete the proof of Main Theorem 1.1 by proving the following result.

Theorem 6.1. The Chow ring $A^{*}\left(\mathcal{M}_{6}^{M}\right)$ is tautological.

For convenience, let $v_{i}:=c_{i}(\mathcal{V})$ (the Chern classes of the Mukai bundle), and $f_{i}:=c_{i}(\mathcal{F})$ (which may be interpreted as pullback of the Chern classes of the tautological rank four vector bundle on $G\left(4, \wedge^{2} \mathcal{V}\right)$, via the section $\mathcal{M}_{6}^{M} \rightarrow G\left(4, \wedge^{2} \mathcal{V}\right)$ to the projection $\left.G\left(4, \wedge^{2} \mathcal{V}\right) \rightarrow \mathcal{M}_{M}^{6}\right)$.

Proposition 6.2. The Chow ring $A^{*}\left(\mathcal{M}_{6}^{M}\right)$ is generated by $f_{1}, \ldots, f_{4}, v_{1}, \ldots, v_{5}$.

Proof. By Theorem 2.3, the Chow ring of $\mathcal{M}_{6}^{M}$ is generated by the Chern classes of $\mathcal{V}$ and (arbitrarily chosen pullbacks of) a basis of the Chow groups of $Y \backslash \Delta$.

Now $Y$ is a vector bundle over $G\left(4, \wedge^{2} V\right)$, so the Chow ring of $Y \backslash \Delta$ is generated by the Chow groups of $G\left(4, \wedge^{2} V\right)$, which are in turn generated as an algebra by the four Chern classes of the tautological bundle of $G\left(4, \wedge^{2} V\right)$. The $f_{i}$ are lifts of these.

Thus to prove Theorem 6.1, it suffices to prove that $f_{1}, \ldots, f_{4}, v_{1}, \ldots, v_{5}$ are tautological. By exact sequence (5.2), the $f_{i}$ lie in $R^{*}\left(v_{1}, \ldots, v_{5}\right)$ (the ring generated by the tautological ring and $\left.v_{1}, \ldots, v_{5}\right)$, so it suffices to prove that $v_{1}, \ldots, v_{5}$ are tautological.

Let $\pi: \mathcal{C} \rightarrow \mathcal{M}_{6}^{M}$ be the universal curve over the Mukai-general locus $\mathcal{M}_{6}^{M}$. Then on $\mathcal{M}_{6}^{M}$, we have an exact sequence

$$
0 \rightarrow \mathcal{G} \rightarrow \operatorname{Sym}^{2} \pi_{*} \omega \rightarrow \pi_{*}\left(\omega^{\otimes 2}\right) \rightarrow 0
$$




\section{The Chow Ring of The Moduli SPACE of CURVES of Genus SIX}

Here $\omega$ is the relative dualizing sheaf as usual, and $\mathcal{G}$ is a rank six vector bundle, which over each point $[C] \in \mathcal{M}_{6}$ corresponds to the six-dimensional vector space of quadrics cutting out the canonical curve $C \subset \mathbb{P} H^{0}\left(C, \omega_{C}\right)$.

Just as in $\S 3.5 .3$, the Chern classes of $\mathrm{Sym}^{2} \pi_{*} \omega=\mathrm{Sym}^{2} \mathbb{E}$ are tautological, and the Chern classes of $\pi_{*} \omega^{\otimes 2}$ are tautological using Grothendieck-Riemann-Roch. Thus the Chern classes of $\mathcal{G}$ are also tautological.

Note that $\mathcal{G}$ has a canonical rank five subbundle corresponding to the five quadrics cutting out the Plücker embedding of $G(2, V)$. In other words, we have an exact sequence

$$
0 \rightarrow \mathcal{V}^{\prime} \rightarrow \mathcal{G} \rightarrow \mathcal{L} \rightarrow 0
$$

where $\mathcal{L}$ is an invertible sheaf. Now $\mathcal{L}$ has tautological Chern class (by Proposition 4.3), so $\mathcal{V}^{\prime}$ has tautological Chern classes.

Lemma 6.3. Suppose $V$ is a dimension five vector space. We have the Plücker embedding $G(2, V) \hookrightarrow \mathbb{P} \wedge^{2} V$, cut out by the five Plücker quadrics. The quadrics are, as a $G L(V)$ representation, $\wedge^{4} V=V^{\vee} \otimes \operatorname{det} V$.

Proof. This is a standard argument. (For example, let $e_{1}, \ldots, e_{5}$ be a basis of $V$. We may explicitly write down the Plücker quadrics in terms of the Plücker variables $e_{i j}:=e_{i} \wedge e_{j}$. Character theory then allows us to determine the $G L(V)$-representation on these quadrics.)

By our construction of $\mathcal{M}_{6}^{M}$ as a $G L(V)$-quotient, we have $\mathcal{V}^{\prime}=\mathcal{V}^{\vee} \otimes \operatorname{det} \mathcal{V}$. As $\operatorname{det} \mathcal{V}$ has tautological Chern class (Proposition 4.3), $\mathcal{V}^{\vee}$ has tautological Chern classes, and hence $\mathcal{V}$ does too.

\section{ACKNOWLEDGEMENTS}

This paper relies in an essential way on the ideas of the first author's Ph.D. thesis at Stanford University (2009). The paper owes an obvious debt to C. Faber, and we are grateful to him. We also thank G. Farkas and S. Grushevsky for helpful conversations. We thank the referee for several important comments and corrections. The second author thanks the American Institute of Mathematics for hospitality during the writing of this article.

\section{REFERENCES}

ACGH85 E. Arbarello, M. Cornalba, P. Griffiths, and J. Harris, Geometry of Algebraic Curves I, Grund. der math. Wiss. 267, Springer, Berlin, 1985.

AH81 E. Arbarello and J. Harris, Canonical curves and quadrics of rank 4, Compos. Math. 43 (1981), no. $2,145-179$.

AV04 A. Arsie and A. Vistoli, Stacks of cyclic covers of projective spaces, Compos. Math. 140 (2004), no. 3, 647-666. http://dx.doi.org/10.1112/S0010437X03000253

BV12 M. Bolognesi and A. Vistoli, Stacks of trigonal curves, Trans. Amer. Math. Soc. 364 (2012), no. 7, 3365-3393. http://dx.doi.org/10.1090/S0002-9947-2012-05370-0

Fab90a C. Faber, Chow rings of moduli spaces of curves I: The Chow ring of $\overline{\mathcal{M}}_{3}$, Ann. of Math. 132 (1990), 331-419.

Fab90b_ Chow rings of moduli spaces of curves II: Some results on the Chow ring of $\overline{\mathcal{M}}_{4}$, Ann. of Math. 132 (1990), 421-449.

Fab97 , A non-vanishing result for the tautological ring of $\mathcal{M}_{g}$, arXiv:math.AG/9711219. 


\section{Nikola Penev and Ravi Vakil}

Fab99 A A conjectural description of the tautological ring of the moduli space of curves, in Moduli of curves and Abelian Varieties, C. Faber and E. Looijenga (eds), 109-129, Aspects of Mathematics E33, Vieweg Wiesbaden, 1999.

FL08 C. Fontanari and E. Looijenga, A perfect stratification of $\mathcal{M}_{g}$ for $g \leqslant 5$, Geom. Dedicata 136 (2008), 133-143.

Ful84 W. Fulton, Intersection Theory, Ergeb. der Math. 2, Springer, Berlin, 1984.

Iza95 E. Izadi, The Chow ring of the moduli space of curves of genus 5, in The Moduli Space of Curves, R. Dijkgraaf, C. Faber, and G. van der Geer (eds), Progress in Math. 129, Birkhäuser, Boston, 1995.

Kre99 A. Kresch, Cycle groups for Artin stacks, Invent. Math. 138 (1999), no. 3, 495-536.

Loo95 E. Looijenga, On the tautological ring of $\mathcal{M}_{g}$, Invent. Math. 121 (1995), no. 2, 411-419.

Muk93 S. Mukai, Curves and Grassmannians, Algebraic Geometry and Related Topics (Inchon, 1992), 19-40, Conf. Proc. Lecture Notes Algebraic Geom., I, Int. Press, Cambridge, MA, 1993.

Mum77_ Stability of projective varieties, Enseignement Math. (2) 23 (1977), no. 1-2, 39-110.

Mum83 D. Mumford, Toward an enumerative geometry of the moduli space of curves, in Arithmetic and Geometry, Vol. II, M. Artin and J. Tate (eds), 271-328, Prog. Math. 36, Birkhäuser, Boston, MA, 1983.

S-D73 B. Saint-Donat, On Petri's analysis of the linear system of quadrics through a canonical curve, Math. Ann. 206 (1973), 157-175.

Sch86 F.-O. Schreyer, Syzygies of canonical curves and special linear series, Math. Ann. 275 (1986), no. 1, 105-137.

Sta00 Z. Stankova-Frenkel, Moduli of trigonal curves, J. Alg. Geom. 9 (2000), no. 4, 607-662.

Vis87 A. Vistoli, Chow groups of quotient varieties, J. Algebra 107 (1987), 410-424.

Wit91 E. Witten, Two-dimensional gravity and intersection theory on moduli space, Surveys in Differential Geometry (Cambridge, MA, 1990), 243-310, Lehigh Univ., Bethlehem, PA, 1991.

Nikola Penev npenev@gmail.com

Ravi Vakil vakil@math.stanford.edu

Department of Mathematics, Stanford University, Stanford, CA 94305, USA 